\title{
Topics
}

\section{Emin Gahramanov* \\ On the Demographics and the Severity of the Social Security Crisis}

DOI 10.1515/bejeap-2015-0098

Published online December 8, 2015

Abstract: Changing demographics across the world threatens the sustainability of pension benefits. Yet there is widespread sentiment among some business and policy analysts that in the presence of population ageing, more elderly people would mean more old-age consumption and robust business opportunities across all spending dimensions. In this paper we look at a micro-level analysis of intertemporal consumption/saving behavior, and find that in the presence of notable heterogeneity with respect to the consumer impatience and rationality degree, different demographic challenges and likely policy responses would imply greatly varying and significant consumption changes at old age. We also touch upon the associated issues of welfare analysis and transitional effects and discuss various complexities and challenges for policy implications and economic projections.

Keywords: pension crisis, perfect foresight, rule of thumb, baby boom, life expectancy

JEL Classification: D91, E21, H55, D6

\section{Introduction}

There is general agreement between economists and policymakers that an ageing phenomenon across the globe would present considerable challenges. In the context of ageing, many academic and policy debates revolve around the solvency of social security and disability programs (see, e.g., Brunner 2002; Lacomba and Lagos 2006), as well as rising healthcare and medicare costs. Yet many business circles view ageing as an open door to exciting opportunities. According to Boyle (2013) at Bloomberg, "silver shoppers" and baby boomers represent trillions of dollars' worth of consumer segment, as elderly “... are looking for new experiences, and two-thirds of them plan to spend more time on hobbies and interests than they do today." Government officials in many countries agree. According to

*Corresponding author: Emin Gahramanov, Department of Economics, American University of Sharjah, University City, Sharjah, United Arab Emirates, E-mail: egahramanov@aus.edu 
the New South Wales Government (Australia), in the next few decades millions “... of seniors will want products and services to suit their lifestyles, creating new markets and business opportunities.” (NSW Government 2012, 17). According to Bradshaw (2015), the Chinese government reports that by 2050 there will be a significant rise of China's consumption related to the elderly, and this business opportunity "... is something to work on immediately."

However, based on a micro-level analysis of intertemporal spending decisions under various demographic phenomena and likely policy responses, we argue that projections of the elderly's future spending patterns would be greatly hindered by a number of complex factors. In the not so distant future, the elderly are likely to live in a very different world of pension provisions. As a result, a given elderly person may actually decide to spend very differently than his predecessors. But this is still not the whole story. Ageing is not a homogeneous phenomenon. Population ageing might be more pronounced due to declining birth rates or due to the retirement of baby boomers or due to increased longevity. Ageing may also be due to a combination of several complex factors. Adding to this complexity is the fact that many consumers may have inherently different attitudes towards life cycle saving and spending, let alone varying unobservable characteristics.

In this paper, we focus on a shortfall of pension benefits and analyze the effect of a baby-boom-and-bust and increased longevity. In the context of these demographic scenarios, we focus on the intertemporal life cycle consumption decisions of perfect-foresight ("rational") plus simple Keynesian rule-of-thumb ("myopic") agents, with special attention to the role of consumer impatience. We further analyze a scenario with a mandatory retirement age adjusted in such a way as to keep the dependency ratio constant. In addition, we consider the transition phase between the steady states. In general, although it may be tempting to think that myopia will result in a greater consumption loss of unprepared rule-of-thumb agents compared to their perfect-foresight brethren, we find this is not always the case. We show the two demographic scenarios have a different impact on consumption decisions and this impact furthermore depends on the assumptions concerning the savings rate of the rule-following agents as well as on possible policy responses to the ageing process. We find that perfect-foresight consumers' relative consumption changes due to an ageing phenomenon can be higher or lower than that of the rule-of-thumb agents depending on the value of the former agents' impatience level. With respect to the transition results, we again observe notable differences in consumption losses that can be severe yet sensitive to the parameterization.

In addition, what is important for business forecasters and policymakers to keep in mind is that in a world of stable factor prices and linear production 
technology and constant labor productivity, the aggregate income (and consumption) is just proportional to the length of the working period. Thus, an increase in the old-age consumption would be, other things being equal, counter-balanced by a drop in young-age consumption. Under various demographic changes and policy responses this may change, yet as can be fairly straightforwardly shown within our framework, aggregate income would still remain the same for both "myopia" and "perfect foresight" scenarios. ${ }^{1}$ Thus, when policymakers make statements concerning consumption, they should be quite cautious about over-emphasizing potential business opportunities, and be aware of the interconnections between spending patterns at various ages. Certainly, even under the constancy of aggregate income there might be a significant impact on sectoral production. This is because younger and old individuals do tend to consume different types of goods, and various studies showed significant heterogeneity in the spending patterns of the elderly and young (particularly in the areas of healthcare, education, and energy). ${ }^{2}$ On the other hand, for some goods and services, there might be very little spending pattern differences at different ages. An example of this might be public transportation in some developed countries where there is growing evidence that many younger people also spend significantly on public transportation (The Rockefeller Foundation 2014). This is important, as many governments do actively seek to invest in public transportation to meet the projected demands of the elderly. In addition, there might also be a link between consumer sophistication and patience on the one hand, and their spending patterns at various ages on the other hand. Therefore, future studies should strive to properly estimate such links under changing demographics and heterogeneity, while carefully relating these intricate micro-level connections to sectoral production at the aggregate level.

Our aim is to also gain insights into welfare analysis. The latter can be particularly relevant for policymakers. Even though in a single period the relative consumption loss may be more severe with a life expectancy-driven crisis, the agent actually lives longer under increased longevity. Thus, his lifetime utility may be larger as there are more periods being summed up. We find that an improvement in welfare is very sensitive to the impatience level, yet may still arise even for a reasonably modest increase in life expectancy. This raises further concerns from the perspectives of the Repugnant Consumption and population ethics. ${ }^{3}$

1 We thank the Editor and an anonymous referee for bringing these relations to our attention. 2 See, e.g., Abdel-Ghany and Sharpe (1997) and Kronenberg (2009).

3 We are thankful to an anonymous referee for drawing our attention to this important issue. 
We next would like to highlight why we are analyzing rational life-cyclers versus myopes, while also paying attention to consumer impatience. First, it is a tradition in orthodox economics to assume that people systematically behave in a rational manner and that consumers engage in consumption-smoothing. However, there is also ample evidence for the prevalence of Keynesian rule-ofthumb consumers (e.g., Campbell and Mankiw 1989, 1990; Thaler 1994; Lusardi 1999; Hurst and Willen 2007; Wong 2012). Further to this, many authors incorporated a rule-based myopic behavior in the quantitative-theoretic assessments of public pension provision and related issues. ${ }^{4}$ In addition, recent studies in behavioral economics, argued that some of the observed rules-of-thumb might actually be "hyperrational", and result from the human evolutionary process and natural selection (Feigenbaum, Caliendo, and Gahramanov 2011; Feigenbaum, Gahramanov, and Tang 2013a, 2013b). We thus note that paying careful attention to various personal characteristics in assessing an effect of an economic factor might be valuable.

Another point we would like to make is that it is known a key element in intertemporal decision making is the value of the individual rate of time preference. There is ample literature suggesting that the discount rate may vary greatly across individuals, and such heterogeneity may explain significant wealth heterogeneity across households, while also being correlated with various individual characteristics (e.g, education, age, gender, or family status). We thus aim to highlight the role of the discount rate in our analysis.

In sum, we suggest both private and public sector analysts pay close attention to and engage in a deeper discussion on the microeconomics behind the elderly's likely intertemporal spending behavior in the context of changing pension systems and population projections. In addition, as there might be connections between spending patterns at different ages and the type of consumer, future research should investigate the interaction of household behavior and resulting sectoral demand at the aggregate level. Although it is virtually impossible in practice to identify an exact type of consumer, knowing the distribution of consumer types within a country would help to optimally plan for the provision of public programs, and forecast creative business opportunities. This necessiates more detailed micro-econometric studies and household surveys. Further, investigating some important paradoxes in population ethics in the context of various demographic and pension provision scenarios presents

4 See, e.g., Feldstein (1985), Docquier (2002), Kaplow (2006), Findley and Caliendo (2007), Cremer et al. (2008), Findley and Caliendo (2010). Findley and Caliendo (2007, 2010), for instance, focus on the rule-based private SMarT saving plan, with longevity being fixed. 
considerable challenges, and in light of this, more research on a socially ethical analyses of heterogenous agents' welfare in the context of ageing is needed.

The rest of this paper is organized as follows. Section 2 presents the main theoretical results. Section 3 presents numerical findings, while Section 4 presents some extensions. The last section presents the conclusion.

\section{Basic Theory}

\subsection{Retirement of Baby Boomers}

We intentionally keep the model as simple as possible. There is no uncertainty, and the framework in question is partial equilibrium. Markets are complete and there are no borrowing constraints. Time $(t)$ is continuous. The agent enters the workforce at time $t=0$, retires at $t=T$, and dies at $t=\bar{T}$. The wage rate is $w$, and the financial asset account, $k(t)$, grows at a market real rate of return, $r$. The social security tax is $\theta$. When retired, the agent receives social security benefits from a pay-as-you-go system, $b=R \theta w$, where $R$ is the worker-to-retiree ratio. Disposable wages exceed retirement benefits.

When a previously born cohort of baby boomers enters the retirement stage, there is a drop in the worker-to-retiree ratio from $R$ to $\tilde{R}$. Thus, we can consider a new steady state with benefits $\tilde{b}=\tilde{R} \theta w$, where $b>\tilde{b}$.

The perfect foresight (" $p f$ " for short) consumer solves the following control problem

$$
\max _{\left\{c^{p f}(t)\right\}} \int_{0}^{\bar{T}} e^{-\rho t} u\left(c^{p f}(t)\right) d t
$$

subject to

$$
\begin{gathered}
\frac{d k(t)}{d t}=r k(t)-c^{p f}(t), \\
k(0)=\int_{0}^{T} e^{-r t}(1-\theta) w d t+\int_{T}^{\bar{T}} e^{-r t} b d t, \\
k(\bar{T})=0 .
\end{gathered}
$$

The utility function takes the form $\frac{\left(c^{p f}(t)\right)^{1-\sigma}}{1-\sigma}$, where $\sigma$ is the inverse elasticity of intertemporal substitution, and the rate of time preference is $\rho$. Hence, application of the Maximum Principle yields the optimal control 


$$
c^{p f}(t)=\frac{k(0) e^{r \bar{T}+g t}(g-r)}{e^{g \bar{T}}-e^{r \bar{T}}},
$$

for $t \in[0, \bar{T}]$, and where $g \equiv(r-\rho) / \sigma$. In a separate steady-state with $\tilde{R}$ workers per retiree, the consumption program is

$$
\tilde{c}^{p f}(t)=\frac{\tilde{k}(0) e^{r \bar{T}+g t}(g-r)}{e^{g \bar{T}}-e^{r \bar{T}}},
$$

where the new initial condition, $\tilde{k}(0)$, is the same as eq. [3] but with benefits equal to $\tilde{b}$.

Before we proceed, we assume that $g \neq r$ to prevent the consumption paths in eqs [5] and [6] being indeterminate. Next, we demonstrate the following proposition.

Proposition 1: When the source of the crisis is the retirement of baby boomers, the percentage change in the consumption of a perfect foresight consumer across the two steady states is invariant to the discount rate, $\rho$, the curvature parameter, $\sigma$, the wage rate, $w$, and the point in time in the life cycle at which the comparison is made.

\section{Proof: See Appendix A.}

Proposition 1 is in line with the traditional theoretical results on the life cycle hypothesis with a fully stationary environment featuring zero interest rate and constant optimal consumption over life. Such environment implies the wealthincome ratio and savings rate are independent from the model's key preference parameters and income level but are dependent on demographic factors (see Modigliani 1986; Piketty 2014). We recall that in an economy with positive growth rates, a fall in the growth rate of the population implies that the social security program features a lower internal rate of return. Note that the internal rate of return (call it $v$ ) of the pension system is determined (at the date of retirement) from

$$
\int_{0}^{T} \theta w e^{v(T-t)} d t=\int_{T}^{\bar{T}} R \theta w e^{\nu(T-t)} d t .
$$

Indeed, using the parameter values from the baseline scenario (see Section 3, Table 1), we find that when $R$ is 3, the internal rate of return is practically zero. Suppose a fall in the population growth rate triggers a fall in the worker-to-retiree ratio, say, to 2 . This would clearly lead to an even smaller value of $v=-1.575 \% .^{5}$

5 We are thankful to an anonymous referee for bringing these relations to our attention. 
Table 1: Baseline parameterization.

\begin{tabular}{llr}
\hline Parameter name & Symbol & Value \\
\hline Date of retirement & $T$ & 40 \\
Date of death & $\bar{T}$ & $53 \frac{1}{3}$ \\
Real rate of return & $r$ & 0.035 \\
Discount rate & $\rho$ & 0.035 \\
Curvature parameter & $\sigma$ & 0.5 \\
Social Security tax rate & $\theta$ & 0.106 \\
Wage rate & $w$ & $\$ 40,000$ \\
\hline
\end{tabular}

We now turn to a separate type of consumer - the rule-of-thumb consumer ("rot", for short) who saves a fixed fraction, $s$, of his disposable wage earnings during his working years, and annuitizes the balance upon retirement. The agent's capital account evolves according to the following differential equations and end-point conditions

$$
\begin{gathered}
\frac{d k(t)}{d t}=r k(t)+s(1-\theta) w \quad \forall t \in[0, T], \\
\frac{d k(t)}{d t}=r k(t)-A \quad \forall t \in[T, \bar{T}], \\
k(0)=0, \\
k(\bar{T})=0,
\end{gathered}
$$

where $0 \% \leq s<100 \%$ is the saving rate, and $A$ is the constant annuity withdrawal that eventually drives the account to zero. We show in Appendix B that

$$
A=\frac{s(1-\theta) w\left(1-e^{r T}\right)}{e^{r(T-\bar{T})}-1} \quad \forall t \in[T, \bar{T}] .
$$

Clearly, in the current steady-state the rule-of-thumb agent's working life consumption is

$$
c^{r o t}(t)=(1-s)(1-\theta) w \quad \forall t \in[0, T],
$$

while his retirement consumption is

$$
c^{r o t}(t)=A+b \quad \forall t \in[T, \bar{T}] .
$$

In the new steady state, his retirement consumption will be

$$
\tilde{c}^{r o t}(t)=A+\tilde{b} \quad \forall t \in[T, \bar{T}],
$$

with $\tilde{c}^{r o t}(t)<c^{r o t}(t)$. 
Proposition 2: When the source of the crisis is the retirement of baby boomers, the percentage change in retirement consumption of the rule-of-thumb consumer across the two steady states is invariant to time, $t$, and the wage rate, $w$, but is positively related to the savings rate, s. Hence, the percentage drop in retirement consumption due to a reduction in social security benefits is not as large when more is saved for retirement.

Proof: See Appendix C.

It should be noted the only drop in consumption experienced by the rule-of-thumb consumer occurs during the retirement stage of life. Thus, if the consumer saved more during their working life, he would accumulate more funds in the asset account, mitigating the adverse impact of the drop in the worker-to-retiree ratio.

A drop in the present value of lifetime resources due to lower pension benefits, causes a change in lifetime utility of the economic agent, ceteris paribus. The severity of the change might be sensitive to model parameters. To assess a relative utility loss we, in line with Findley and Caliendo (2009), first state the following definition.

Definition: The "compensating variation" is the constant fraction of consumption that has to be given to a consumer who lives in a steady state with lower pension benefits in order to equate his lifetime utility with the utility of an identical consumer receiving higher pension benefits in a different steady state.

Note that in this section we will deliberately limit our attention to the welfare analysis of a perfect-foresight consumer, since welfare comparisons involving a non-optimizing consumer would not be straightforward. The latter issue is further discussed in Section 4.1.

For the perfect foresight consumer, the compensating variation, $\varsigma^{p f}$, solves

$$
\int_{0}^{\bar{T}} e^{-\rho t} \frac{\left(c^{p f}(t)\right)^{1-\sigma}}{1-\sigma} d t=\int_{0}^{\bar{T}} e^{-\rho t} \frac{\left(\left(1+\varsigma^{p f}\right) \tilde{c}^{p f}(t)\right)^{1-\sigma}}{1-\sigma} d t
$$

It follows that

$$
\varsigma^{p f}=\frac{k(0)-\tilde{k}(0)}{\tilde{k}(0)}=\frac{\theta(\tilde{R}-R)\left(e^{-r T}-e^{-r \bar{T}}\right)}{(1-\theta)\left(e^{-r T}-1\right)+\tilde{R} \theta\left(e^{-r \bar{T}}-e^{-r T}\right)} .
$$

Since $k(0)>\tilde{k}(0), \varsigma^{p f}$ is always positive, which is sensible. 
Proposition 3: When the source of the crisis is the retirement of baby boomers, the relative welfare loss of the perfect foresight consumer is invariant to the discount rate, $\rho$. Proof: Refer to eq. [16] and observe that $\partial \varsigma^{p f} / \partial \rho=0$.

The natural question now to ask is whether there is a critical savings rate, $s$, which would make the rule-of-thumb consumer as disadvantaged as the perfect foresight consumer in terms of the relative loss in consumption. The answer critically depends on the source of the pension crisis as can be seen from the following proposition and the subsequent sections.

Proposition 4: When the source of the crisis is the retirement of baby boomers, then regardless of the value of the curvature parameter, $\sigma$, the relative consumption losses of the rule-of-thumb and perfect foresight consumers will not be equal to each other (unless the saving rate is assumed to be $s=100 \%$ ).

Proof: Using eqs [11] and [41], the equality with eq. [35] occurs only when $s$ equals unity.

When the rule-of-thumb consumer saves his entire disposable wage throughout his working life, his consumption loss during retirement is exactly equal to that of the perfect foresight agent. If we recall that the relative consumption drop for the myopic agent must monotonically decline with the saving rate, obviously a ruleof-thumb consumer cannot experience a lower percentage decline in consumption than a perfect foresight consumer. This would imply that the pension crisis for the myopic agent is financially less severe.

\subsection{Higher Life Expectancy vs. Retirement of Baby Boomers}

We now will consider a pension crisis solely driven by an increase in life expectancy. To capture the life expectancy-driven crisis, we assume a finite population spread uniformly from 0 to $\bar{T}$ years. All people die at $\bar{T}$, and when someone dies another person is born. Consequently, $R=T /(\bar{T}-T)$. With higher life expectancy, the finite population becomes spread from 0 to $\bar{F}$ years $(\bar{F}>\bar{T})$. Thus, $\tilde{R}=T /(\bar{F}-T)<R$.

Then, the optimal consumption profile becomes

$$
\tilde{c}^{p f}(t)=\frac{\varkappa(0) e^{r \bar{F}+g t}(g-r)}{e^{g \bar{F}}-e^{r \bar{F}}},
$$

where 


$$
\varkappa(0)=\int_{0}^{T} e^{-r t}(1-\theta) w d t+\int_{T}^{\bar{F}} e^{-r t} \tilde{b} d t .
$$

From eqs [17] and [5], the relative consumption loss of the perfect foresight agent is

$$
\begin{aligned}
\frac{\Delta c^{p f}(t)}{c^{p f}(t)}= & \frac{\varkappa(0)\left(e^{(g-r) \bar{T}}-1\right)}{k(0)\left(e^{(g-r) \bar{F}}-1\right)}-1 \\
= & \left(\frac{(1-\theta)\left(e^{-r T}-1\right)+\tilde{R} \theta\left(e^{-r \bar{F}}-e^{-r T}\right)}{(1-\theta)\left(e^{-r T}-1\right)+R \theta\left(e^{-r \bar{T}}-e^{-r T}\right)}\right) \\
& \times\left(\frac{e^{(g-r) \bar{T}}-1}{e^{(g-r) \bar{F}}-1}\right)-1 .
\end{aligned}
$$

Expression [19] leads to the following proposition.

Proposition 5: When the source of the Social Security crisis is increased life expectancy, the percentage drop in the consumption of perfect foresight agents is less severe when the discount rate is higher.

Proof: See Appendix D.

According to Proposition 5, when higher longevity leads to the pension crisis, a perfect foresight agent with a higher discount rate will experience a relatively lower drop in lifetime consumption (during $t \in[0, \bar{T}<\bar{F}]$, of course) than an otherwise identical agent with a smaller discount rate. The larger the discount rate is, the bigger the absolute level of consumption in the early years will be for any given level of lifetime wealth. A given percentage decline in the consumption path will therefore be larger in absolute magnitude in the early years, which the agent with a higher discount rate is less inclined to tolerate. Thus, such an agent chooses to have quite a low consumption level in very old age rather than in their relatively earlier years. But why doesn't the discount rate play a role when the Social Security crisis is driven solely by the baby boom phenomenon? If we recall that in the case of a baby boom, i.e., when the lifetime does not increase from $\bar{T}$ onward, there is simply no way to have different percentage drops across different perfect foresight agents as this would have implied a more impatient agent's lifetime wealth is larger than that of a less impatient agent. But when a more impatient consumer is allowed to live longer, he can set his consumption from $\bar{T}$ to $\bar{F}$ at the sufficiently low absolute level. 
For the rule-of-thumb consumer, the new annuity withdrawal is

$$
\tilde{A}=\frac{s(1-\theta) w\left(1-e^{r T}\right)}{e^{r(T-\bar{F})}-1} \quad \forall t \in[T, \bar{F}],
$$

and the corresponding relative change in consumption is as follows

$$
\frac{\Delta c^{r o t}(t)}{c^{r o t}(t)}=\frac{\tilde{A}-A+\theta w(\tilde{R}-R)}{A+R \theta w} .
$$

We next establish the following two propositions.

Proposition 6: Regardless of the value of the curvature parameter, $\sigma$, percentage losses in consumption of the rule-of-thumb consumer are equal to those of the perfect foresight consumer if $s=\frac{\theta\left(R a_{4}-\tilde{R}\right)}{a_{5}-a_{4} a_{6}}$, where $a_{5} \equiv \frac{(1-\theta)\left(1-e^{r T}\right)}{e^{r(T-\bar{F})}-1}, a_{6} \equiv \frac{(1-\theta)\left(1-e^{r T}\right)}{e^{r(T-\bar{T})}-1}$, and $a_{4} \equiv\left(\frac{(1-\theta)\left(e^{-r T}-1\right)+\tilde{R} \theta\left(e^{-r \bar{F}}-e^{-r T}\right)}{(1-\theta)\left(e^{-r T}-1\right)+R \theta\left(e^{-r \bar{T}}-e^{-r T}\right)}\right) \times\left(\frac{e^{(g-r) \bar{T}}-1}{e^{(g-r) \bar{F}}-1}\right)$.

Proof: See Appendix E.

Proposition 7: When the source of the Social Security crisis is the retirement of baby boomers, the relative loss in consumption (up to year $\bar{T}$ ) for both perfect foresight and rule-of-thumb consumers is less severe (when $g=0$, and $s>0 \%$, respectively) than what it would be under a longevity-driven crisis. When $g \neq 0$, it is unclear which type of crisis will bring a bigger consumption loss. When $s=0 \%$, the relative loss in consumption for rule-of-thumb consumers under both sources of the crisis is equal.

Proof: See Appendix F.

Intuitively, when a perfect foresight agent with a flat wage-consumption profile lives longer, he must smooth out his wage income over a longer horizon, and this will lead to a reduction in lifetime consumption (provided that wage income exceeds social security benefits, which is true by assumption). Thus, the consumption loss would be even more severe during a single period. However, with a nonflat consumption profile $(g \neq 0)$, the agent may shift his consumption loss to earlier or later stages in life. Proposition 7 also implies that when the source of the crisis is increased life expectancy, the myopic consumer will always suffer a bigger drop in retirement consumption. The key is when everyone lives longer, the rule of thumb consumer in question has a lower private annuity and lower benefits, whereas in the case of the baby-boom phenomenon, he only has lower benefits with the same private annuity. The lower annuity effect obviously vanishes when $s=0 \%$. 
Remark 1: As far as the loss in lifetime utility of the rational agent is concerned, it may be that the lifetime utility loss is not as severe. Or, perhaps the lifetime utility change could even be positive. In a single period the relative consumption loss may be more severe with a life expectancy-driven crisis. On the other hand, when the agent lives longer, his lifetime utility will tend to be larger because there are more periods being added up.

Precisely, the compensating variation for the perfect foresight consumer is now

$$
\begin{aligned}
\varsigma^{p f}= & \left(\frac{(1-\theta)\left(1-e^{-r T}\right)+R \theta\left(e^{-r T}-e^{-r \bar{T}}\right)}{(1-\theta)\left(1-e^{-r T}\right)+\tilde{R} \theta\left(e^{-r T}-e^{-r \bar{F}}\right)}\right) \\
& \times\left(\frac{e^{(g-r) \bar{F}}-1}{e^{(g-r) \bar{T}}-1}\right)\left(\frac{e^{(g(1-\sigma)-\rho) \bar{T}}-1}{e^{(g(1-\sigma)-\rho) \bar{F}}-1}\right)^{\frac{1}{1-\sigma}}-1 .
\end{aligned}
$$

Since comparative statics on eq. [22] with respect to the discount rate produce rather cumbersome results, this time we render them to numerical demonstrations. Nevertheless, a key conclusion from eq. [22] is that a change in discount rate does affect compensating variation for perfect foresight consumers. This is because when the crisis involves everyone living longer than previously, it is relatively less valuable for consumers with a higher psychological discount rate to collect benefits for a longer time.

A word of caution is needed here. Based on Parfit's idea of Repugnant Conclusion, it is clear that population ageing phenomena manifested in increasing life expectancy and different population sizes would make welfare comparisons a delicate ethical issue. It is problematic from an ethical viewpoint to argue that a drop in the quality of life can be compensated by a gain in the population size or even length of life. There has been voluminous literature stating that avoiding the Repugnant Conclusion is not straightforward, and experts in population ethics have long debated how to make consistent welfare comparisons in such circumstances. Consider, for instance, switching to some kind of "average utilitarianism" by deflating the total lifetime welfare of an agent by his corresponding length of life. However, suppose in the new steady state with lower pension benefits and greater longevity we have an agent whose average utility is just slightly lower than that in the old steady state with higher pension benefits. Is it necessarily better to have somebody live a shorter life in just a slightly better condition? Alternatively, in line with "critical level utilitarianism", one might argue that the steady state with lower pension benefits is more desirable only if the per period consumption level does not drop below a certain critical threshold, below which a resulting utility is negative. Yet how to determine such threshold? 
Even if we determined the threshold above some individually neutral level, one could have run into a situation where a greater number of per-period consumptions (before a pension crisis) just below that threshold level but above the neutral level would have lead to more negative welfare values than a smaller number of post-crisis per-period consumptions (perhaps at very old ages) that are below the neutral level. This might lead to the Very Sadistic Conclusion (see Blackorby, Bossert, and Donaldson 2005; Huseby 2012). Although our paper does not aim to tackle the above problems, future research is needed to investigate possible avenues of avoiding potentially unethical conclusions.

\section{Numerical Illustrations}

We now simulate three separate scenarios: (1) the crisis is only triggered by the baby-boom phenomenon; (2) the crisis is only triggered by higher life expectancies; and (3) the crisis is triggered jointly by (1) and (2). Table 1 shows the model parameters.

Let the model age $t=0$ correspond to the actual age of 25, i.e., $T=40$ corresponds to the retirement age of 65 . The total Old-Age and Survivors Insurance tax rate is $10.6 \%$, and thus we set $\theta=0.106$, assuming that labor bears the full burden of the tax. Feigenbaum (2008) considers the curvature parameter $\sigma$ in the vicinity of 0.5 , while setting the interest rate target to $r=0.035$, and thus we do likewise. For simplicity, assume the discount rate is equal to the interest rate, however we do vary it as seen below. We choose $\bar{T}=53 \frac{1}{3}$ in the baseline state. We do this because the uniform population distribution comfortably generates the current ratio of workers to retirees equal to 3 .

Figure 1 plots the percentage losses in consumption of the perfect foresight and rule-of-thumb consumer for various working-age saving rates, $s$, and also the compensating variation of the perfect foresight agent. A lower value of the worker-to-retiree ratio is set at $\tilde{R}=2$.

The loss in consumption of the perfect foresight agent is $1.39 \%$, while the compensating variation is $1.41 \%$ irrespective of the discount rate. Now, let us refer to the scenario where greater longevity is the sole contributor to a drop in retirement benefits, which corresponds to $\bar{F}=60$ (again, $\tilde{R}=2$ ). Figure 2 reports relative changes in consumption and welfare.

If we set the discount rate, for example, to 6\% (Figure 2(a)), the perfect foresight consumer will experience, for compatible years, only $0.88 \%$ lower consumption at each point across the life cycle when we compare a world with three workers per retiree to a world with two workers per retiree. On the other 
a) Consumption Loss: $\rho$ is irrelevant

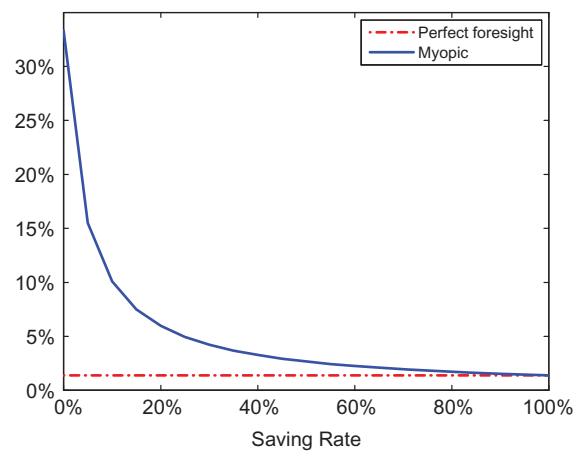

b) Welfare Loss: $\rho$ is irrelevant

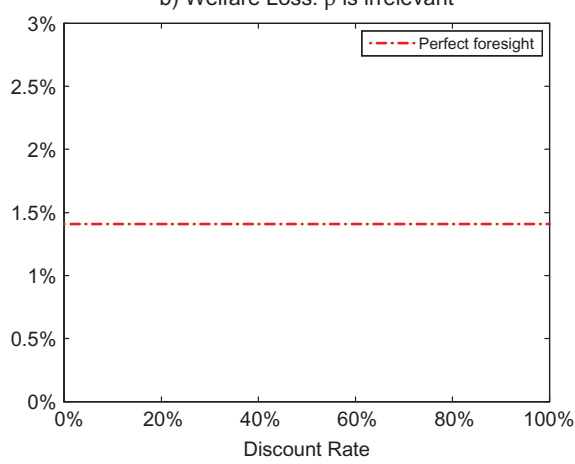

Figure 1: Consumption and welfare changes due to a baby boom-driven crisis.

a) Consumption Loss: $\rho=6 \%$

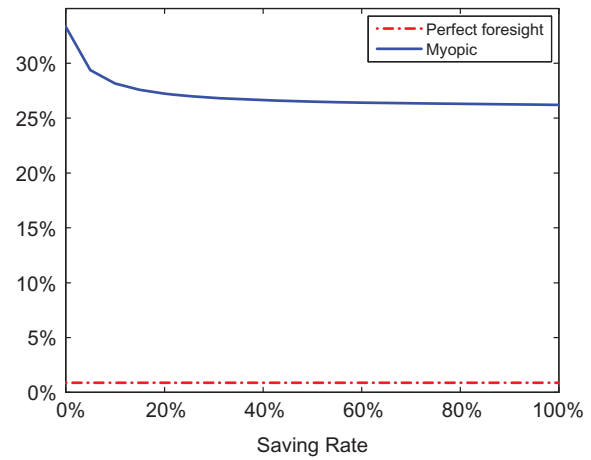

b) Welfare Gain: $\rho$ is relevant

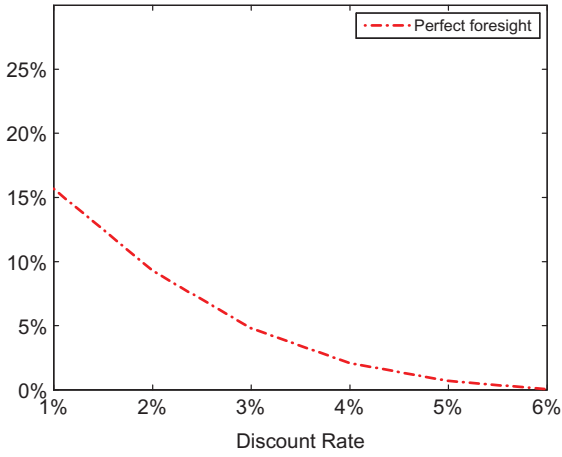

Figure 2: Consumption and welfare changes due to a life expectancy-driven crisis.

hand, the myopic consumer still experiences severe consumption losses during the retirement phase of life. From Figure 2(b), it can be seen that the welfare effect of the rise in dependency ratio is positive for the perfect-foresight agent, yet the higher the discount rate, the smaller the welfare gain.

\subsection{A "Mixed Scenario": Higher Longevity Coupled with the Baby-Boom Effect}

To draw more realistic quantitative results, one needs to recognize that often a fall in the ratio of workers-to-retirees is triggered simultaneously by the 
retirement of baby boomers and everyone living longer. A simple way to demonstrate the implications for such a scenario is to refer to the previous section. It should be noted that a new date of death can be set at some intermediate level between $\bar{T}=53 \frac{1}{3}$ and $\bar{F}=60$, while a further drop in the ratio of workers to retirees can be attributed by the sudden retirement of a large number of baby boomers. We can set the new death date, $\bar{F}$, to 57 , which means the average life expectancy is up to 82 years old. ${ }^{6}$ A further decline in $R$ will thus be assumed to coincide with the population bubble phenomenon. Our simulation results can be summarized as follows.

1) The decline of the myopic consumer's consumption is much smaller but still high (not lower than $17.5 \%$ ) even with a moderate increase in longevity and high saving rates.

2) The drop in consumption of a perfect foresight agent varies greatly. It ranges from $10.06 \%$ (when $\rho=1 \%$ ) to just $1.11 \%$ (when $\rho=6 \%$ ).

3) The lifetime welfare of rational agents improves when the discount rate is relatively low. However, when the discount rate gets even moderately high, the perfect foresight agent suffers a utility loss.

\section{Extensions}

\subsection{Welfare Comparisons in the Presence of Rule-of-Thumb Agents}

In earlier sections we focused on the welfare estimations for rational agents only. Technically, we could have assessed the intertemporal utility of the rule-of-thumb consumers by that of perfect foresight agents as well. Although such an approach would have been in line with some behavioral studies of pension systems (e.g., Feldstein 1985; Docquier 2002; İmrohoroğlu, İmrohoroğlu, and Joines 2003; Hurst and Willen 2007; Cremer et al. 2008), it is certainly controversial. Given that individuals are not maximizing this objective, taking lifetime utility as the benchmark would raise many objections. ${ }^{7}$

In this section, we consider an alternative approach. Let us look at the case where a steady lifetime consumption is the result of an optimizing model (when $r=\rho$, implying $g=0$ ), and the tax rate $\theta$ is such that retirement consumption is

6 This number is close to the intermediate projections for females and males reported by Trustees in Diamond and Orszag (2005).

7 We thank the Editor and an anonymous referee for bringing this point to our attention. 
also the same across two different agents. Thus, using expression [5] we can express a constant consumption profile (call it $c_{\text {const }}^{p f}(t)$ ) as the outcome of the optimizing model:

$$
c_{\text {const }}^{p f}(t)=\frac{k(0) e^{r \bar{T}} r}{e^{r \bar{T}}-1}=\frac{e^{-r T} w\left(e^{r(T+\bar{T})}(1-\theta)-R \theta e^{r T}-e^{r \bar{T}}(1-\theta-R \theta)\right)}{e^{r \bar{T}}-1},
$$

where the tax rate shall be chosen so that eq. [23] equals eq. [13]. After some algebra, we can show that such a social security tax rate must be equal to

$$
\theta=\frac{e^{r \bar{T}}-e^{r T}(1-s)-e^{r(T+\bar{T})} s}{e^{r \bar{T}}(1+R)-e^{r(T+\bar{T})} s-e^{r T}(1+R-s)} .
$$

Substituting eq. [24] into eq. [23], we obtain

$$
c_{\text {const }}^{p f}(t)=\frac{R w(1-s)\left(e^{r T}-e^{r \bar{T}}\right)}{-e^{r \bar{T}}(1+R)+e^{r T}(1+R-s)+e^{r(T+\bar{T})} s} .
$$

Expression [25] is a function of a constant saving rate, s, so that for any value of $s$, there is a flat, perfect-foresight consumption path, where the corresponding consumption rate is equal to that of the rule-of-thumb agent with the saving rate $s$. Consequently, we obtain the percentage change in consumption due to a rise in the dependency ratio:

$$
\frac{\Delta c_{\text {const }}^{p f}(t)}{c_{\text {const }}^{p f}(t)}=\frac{(R-\tilde{R})\left(e^{r \bar{T}}+e^{r T}(s-1)-e^{r(T+\bar{T})} s\right)}{R\left(-e^{r \bar{T}}(1+\tilde{R})+e^{r T}(1+\tilde{R}-s)+e^{r(T+\bar{T})} s\right)} .
$$

So, the optimizing, "constant saving-rule" consumer's compensating variation $\left(s_{\text {const }}^{p f}\right)$ is

$$
S_{\text {const }}^{p f}=\frac{(R-\tilde{R})\left(e^{r \bar{T}}+e^{r T}(s-1)-e^{r(T+\bar{T})} s\right)}{\tilde{R}\left(e^{r \bar{T}}(1+R)-e^{r(T+\bar{T})} s+e^{r T}(s-R-1)\right)} .
$$

Figure 3 plots the compensating variation, against a range of possible saving rates. $^{8}$

We see that a higher (optimal, yet constant) saving rule implies the utility loss is lower due to a shortfall of pension benefits. Yet an important implication for welfare analysis is that seemingly myopic rule-of-thumb behavior can in fact

8 We can evaluate eq. [27] for various saving rates. However, for every saving rate, the payroll tax rate given by eq. [24] gets automatically updated. These different tax rates for the different steady-state comparisons are not problematic because we would keep the tax rates constant across the steady states. Since $\theta$ should not fall outside the $0 \%$ and $100 \%$ region, we have to limit the range of the saving rate, $s$, we can use. 


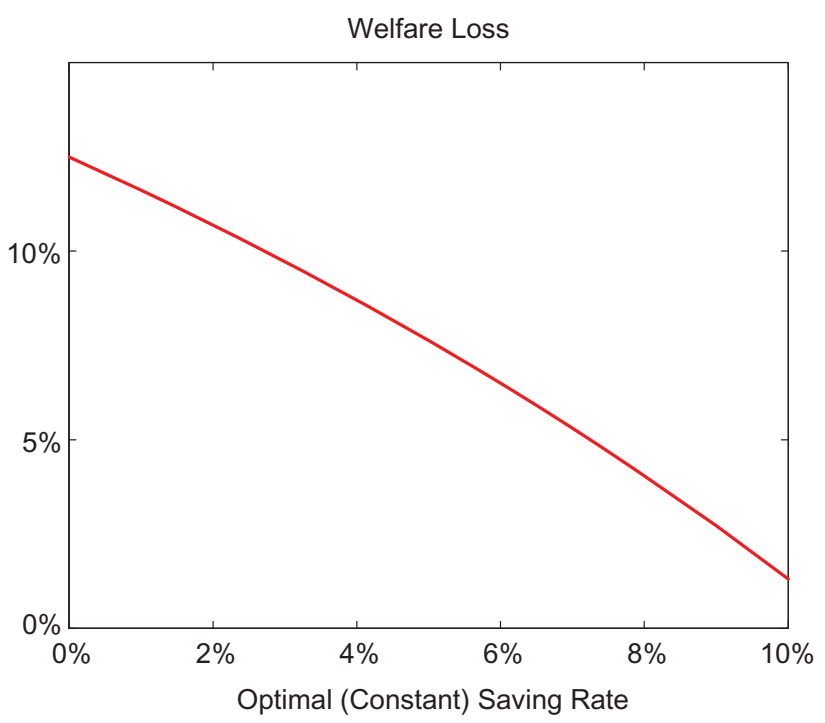

Figure 3: Welfare changes of optimizing "constant saving-rule” agents.

be an outcome of a well-defined (however unconventional) optimizing model. Thus, more empirical research is needed to determine the realism of the objective function in such models. Doing so might greatly aid researchers to undertake consistent and plausible welfare analysis.

\subsection{Cases of Changing Cohort Sizes and Retirement Ages}

In this section, we look at a scenario with changing cohort sizes, and we also consider retirement age increases in such a way as to hold the dependency ratio constant despite higher life expectancy. ${ }^{9}$ We start by assuming that at each instant in time a new cohort replaces an old cohort, yet the size of each cohort grows exponentially at the rate $n>0$. It is straightforward to show that the dependency ratio will depend on $n$ (see also Piketty 2014 on this), and so long as $n$ is constant, the worker-to-retiree ratio will also be constant.

We can further find that for any higher life length $\bar{F}(\bar{F}>\bar{T})$, the retirement date, which ensures the same worker-to-retiree ratio as before (call it $F$ ), is given by

9 We thank an anonymous referee for suggesting to investigate this scenario. 


$$
F=\bar{F} \frac{T}{\bar{T}}
$$

Consequently, the present value of lifetime income for the perfect foresight agent becomes

$$
\varkappa(0)^{n e w}=\int_{0}^{F} e^{-r t}(1-\theta) w d t+\int_{F}^{\bar{F}} e^{-r t} b d t,
$$

where superscript "new" indicates the situation, where an increase in longevity is accompanied by an increase in the retirement age from $T$ to $F=\bar{F} T / \bar{T}$, so that still $b=R \theta w$. Thus, the corresponding consumption profile becomes

$$
\tilde{c}^{p f}(t)^{n e w}=\frac{\varkappa(0)^{n e w} e^{r \bar{F}+g t}(g-r)}{e^{g \bar{F}}-e^{r \bar{F}}} .
$$

From eqs [30] and [5], the relative consumption change of the perfect foresight agent is

$$
\begin{aligned}
\frac{\Delta c^{p f}(t)}{c^{p f}(t)}= & \frac{\varkappa(0)^{n e w}\left(e^{(g-r) \bar{T}}-1\right)}{k(0)\left(e^{(g-r) \bar{F}}-1\right)}-1 \\
= & \left(\frac{(1-\theta)\left(e^{-r F}-1\right)+R \theta\left(e^{-r \bar{F}}-e^{-r F}\right)}{(1-\theta)\left(e^{-r T}-1\right)+R \theta\left(e^{-r \bar{T}}-e^{-r T}\right)}\right) \\
& \times\left(\frac{e^{(g-r) \bar{T}}-1}{e^{(g-r) \bar{F}}-1}\right)-1 .
\end{aligned}
$$

Proposition 8: $\varkappa(0)^{\text {new }}>k(0)$.

Proof: See Appendix G.

However, it is still not clear whether eq. [31] is positive because term $e^{(g-r) \bar{T}}-1$ is less in an absolute sense than term $e^{(g-r) \bar{F}}-1$. Let us suppose $r=0.035, \sigma=1.1, \rho=0.02$, $\theta=0.106, \quad w=1, \quad \bar{T}=53 \frac{1}{3}, \quad \bar{F}=57, \quad T=40$. Thus, $R=3, \quad F=42.75$. Clearly, $\tilde{R}=F /(\bar{F}-F)=3=R$, i.e., $\quad \tilde{b}=b$. As a result, $\quad \varkappa(0)^{\text {new }}=20.6212$, while $k(0)=20.0796$. We obtain $c^{p f}(t)=0.630854 e^{0.0136364 t}$, and $\tilde{c}^{p f}(t)^{\text {new }}=$ $0.625685 e^{0.0136364 t}$, respectively. Thus, we observe a decline in the lifetime consumption path for comparable ages. However, let us raise the discount rate to $\rho=0.03$. Then $c^{p f}(t)=0.761595 e^{0.00454545 t}$, and $\tilde{c}^{p f}(t)^{n e w}=0.762369 e^{0.00454545 t}$, respectively. Thus, we observe a rise in the lifetime consumption path. Intuitively, when the discount rate is relatively high, consumption in older age is low anyway, meaning that an extra gain in the years of life at old age does not require many resources to support late life consumption, thus allowing greater consumption overall. 
Remark 2: Applying the reasonings used in Appendix D, it is not difficult to deduce from eq. [31], that the percentage drop in the consumption of perfect foresight agents would be less pronounced when the discount rate is higher.

Similarly, we can obtain the new annuity withdrawal for the rule-of-thumb agent as

$$
\tilde{A}^{\text {new }}=\frac{s(1-\theta) w\left(1-e^{r F}\right)}{e^{r(F-\bar{F})}-1} \quad \forall t \in[F, \bar{F}],
$$

and the corresponding relative change in post-retirement consumption is as follows

$$
\frac{\Delta c^{r o t}(t)}{c^{r o t}(t)}=\frac{\tilde{A}^{\text {new }}-A}{A+R \theta w} .
$$

Proposition 9: $\tilde{A}^{\text {new }}>A$, implying that when the longevity increase is associated with a sufficient rise in the retirement age so the dependency ratio is held constant, the rule-of-thumb agents' consumption actually increases.

Proof: See Appendix H.

Intuitively, continuous compounding provides exponential growth of the myopic agent's financial account over a longer period of working life, and when the agent finally retires, he can afford greater annuity amount withdrawal over a somewhat longer retirement span. Thus, $\tilde{A}^{\text {new }}>A$, and because the same amount of pension benefit is now preserved over the retirement span, the rule-of-thumb agent's retirement consumption is greater.

Given that our myopic agent unequivocally obtains a gain in his post-retirement consumption under the present scenario, let us see what the discount rate of the perfect-foresight consumer should be so the latter receives at least the same consumption gain. Suppose the saving rate, $s$, is equal to $3.5 \%$, which is consistent with the estimates in Thaler and Benartzi (2004). Using the same parameter values as in Table 1, as well as $\bar{F}=57$ and $F=42.75$, we plot the percentage gain in the consumption of myopic agents, and the percentage changes of lifetime consumption of rational agents under different discount rates.

When the saving rate is mildly high, no matter how impatient the rational agent is, his maximum consumption gain is lower than that of the myopic agent. Yet if we drop $s$ to $1 \%$, relatively impatient rational agents experience bigger consumption gain. Figure 4 also shows rational agents with very low discount rate will actually experience a big consumption loss. 


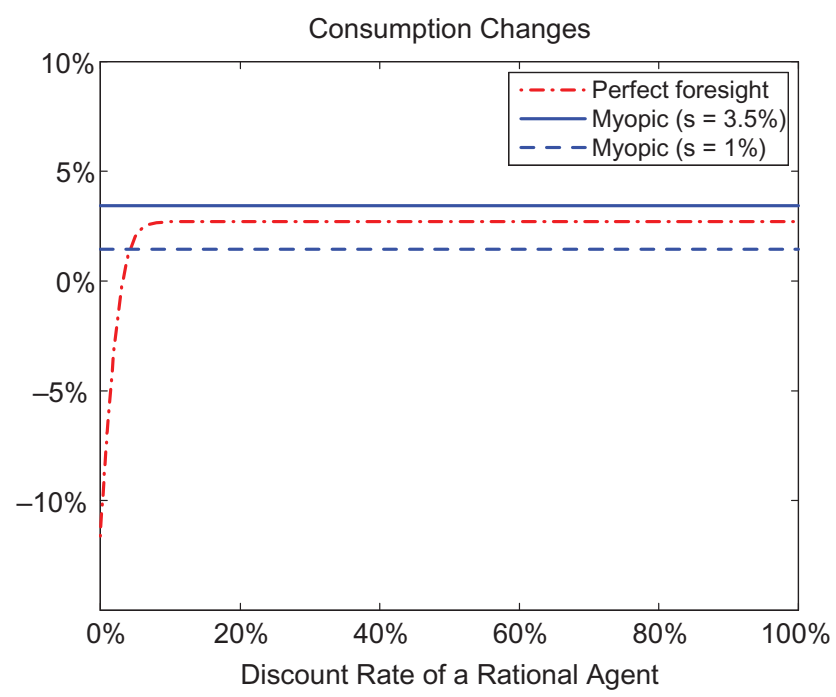

Figure 4: Consumption changes due to a higher life expectancy but later retirement.

\subsection{Transition Phase}

In this section, we briefly look at the transition between the steady states. Suppose a demographic change occurs at time $t=t_{0}$, and it takes the economy $X>0$ years to settle at the new steady state. It would be particularly interesting to compare and contrast the retirement consumption rates of various agents (who just retired at time $t=t_{0}$ ), as well as those agents who enter the model on and after time $t=t_{0} .{ }^{10}$ Table 2 reports the range of the consumption changes (for comparable years of lifetime) of the two types of agents who were born during the first twenty five years of the transition under various scenarios. ${ }^{11}$ Table 3 reports the retirement consumption changes of the agents who are just retired.

It can be clearly seen that consumption losses can be quite pronounced, and vary according to the type of agent. These losses are particularly pronounced for the agents who are just retired at the time of a demographic change. In general,

10 The full analysis and computational details are found in a technical appendix available upon request.

11 For example, the range $[-4.6 \% ;-3.9 \%]$ in the third column $(B B+L E)$, second raw (Rule-ofThumb) means that the rule-of-thumb agents, born during the first twenty five years of transition, on average suffer (relative to the benchmark steady state) consumption losses between 4.6 and $3.9 \%$. " $B B+L E$ " means the rise in the dependency ratio is triggered jointly by the babyboom-and-bust and greater life expectancy. 
Table 2: Consumption changes for those born after the shock, $\rho=3.5 \%, s=3.5 \%$.

\begin{tabular}{lrrrr}
\hline & $B B$ only & LE only & BB+LE & LE+High Ret. \\
\hline Perfect-foresight & {$[-5.7 \% ;-4.3 \%]$} & {$[-7.5 \% ;-6.4 \%]$} & {$[-6.8 \% ;-5.5 \%]$} & {$[-3.0 \% ;-1.9 \%]$} \\
Rule-of-Thumb & {$[-3.0 \% ;-2.3 \%]$} & {$[-5.5 \% ;-4.8 \%]$} & {$[-4.6 \% ;-3.9 \%]$} & {$[-4.4 \% ; 5.0 \%]$} \\
\hline
\end{tabular}

Table 3: Consumption changes for those who are just retired at the time of the shock, $\rho=3.5 \%$, $s=3.5 \%$.

\begin{tabular}{lcccr}
\hline & BB only & LE only & BB+LE & LE+High Ret. \\
\hline Perfect-foresight & $-13.0 \%$ & $-28.0 \%$ & $-22.8 \%$ & $-9.8 \%$ \\
Rule-of-Thumb & $-18.3 \%$ & $-30.0 \%$ & $-25.9 \%$ & $-7.2 \%$ \\
\hline
\end{tabular}

we find the magnitude of the changes are quite sensitive to the parameterization used, meaning that a standard steady state comparison should be contrasted with a more complicated transition analysis. Doing so would enable a richer set of results to be obtained, yet it would certainly add an additional layer of analytical and computational complexity in trying to project future consumption patterns for the elderly in the immediate, as well as distant future.

\section{Conclusion}

There is widespread sentiment among business and policy analysts that given population ageing, more elderly people would mean more old-age consumption, and thus huge business opportunities. In this paper, we look at a micro-level analysis of intertemporal consumption/saving behavior, and find that in the presence of heterogeneity with respect to the levels of impatience, as well as how closely consumers follow a neoclassical model of spending, different demographic challenges might imply greatly varied consumption changes at old age. For instance, we find that a baby-boom-and-bust phenomenon would generally lead to significant consumption losses for rule-of-thumb consumers, and these losses can be assumed to be low only when an individual savings rate is very high. In addition, while in a baby-boom-and-bust phenomenon, impatience heterogeneity plays no role in quantifying a perfect-foresight consumer's spending changes, however it does so if one assumes an increase in longevity. For example, if one assumes everyone is very impatient, then a shortfall in pension benefits would lead to a moderate decline in per-period consumption of a rational agent, yet still leads to very low spending levels at very old age. In contrast, a rule-of- 
thumb consumer would suffer larger consumption losses. However, these losses would turn into gains if one models a policy response where an increase in longevity is matched by a proportional increase in the retirement age in order to preserve the current benefit levels. But if so, perfect foresight consumers would experience, for many years during the old age, a big consumption loss or moderate consumption gain depending on how impatient they are. We also touch upon the issues of welfare analysis and transitional comparisons, and discuss the associated complexities and challenges. We suggest future research also focus on the interaction of household behavior and resulting sectoral demand at the aggregate level. We hope by identifying (via surveys and empirical studies) the distribution of the core consumer types, accounting for some of the key behavioral and psychological differences among the consumers, while focusing on their micro-level decisions in the context of demographic phenomena, will aid policymakers and business circles in their future projections.

Acknowledgments: I thank Johann Brunner and two anonymous referees for their very helpful comments. I would also like to thank Frank Caliendo and Scott Findley for many helpful discussions and encouragement. All errors are my own.

\section{Appendix A}

Using eqs [5] and [6], we obtain the percentage change in consumption of the perfect foresight agent:

$$
\frac{\Delta c^{p f}(t)}{c^{p f}(t)} \equiv \frac{\tilde{c}^{p f}(t)-c^{p f}(t)}{c^{p f}(t)}=\frac{\tilde{k}(0)-k(0)}{k(0)} .
$$

Integrate out $k(0)$ and $\tilde{k}(0)$, and substitute into eq. [34] to get

$$
\frac{\theta(\tilde{R}-R)\left(e^{-r \bar{T}}-e^{-r T}\right)}{(1-\theta)\left(e^{-r T}-1\right)+R \theta\left(e^{-r \bar{T}}-e^{-r T}\right)} .
$$

Expression [35] is invariant to $\rho, \sigma, w$ and $t$.

\section{Appendix B}

The general solution to differential equation [7] is

$$
k(t)=-\frac{s(1-\theta) w}{r}+z_{1} e^{r t},
$$


where $z_{1}$ is constant. Using the initial solution $k(0)=0$, a particular solution is

$$
k(t)=\frac{s(1-\theta) w}{r}\left(e^{r t}-1\right)
$$

which implies

$$
k(T)=\frac{s(1-\theta) w}{r}\left(e^{r T}-1\right) .
$$

The general solution to differential equation [8] is

$$
k(t)=\frac{A}{r}+z_{2} e^{r t},
$$

where $z_{2}$ is constant. Using the boundary condition $k(\bar{T})=0$, a particular solution is

$$
k(t)=\frac{A}{r}\left(1-e^{r(t-\bar{T})}\right) .
$$

Finally, we obtain eq. [11] by setting $t$ equal to $T$ in eq. [40], and then equating eqs [40] and [38] to solve for $A$.

\section{Appendix C}

Using eqs [13] and [14], we obtain

$$
\frac{\Delta c^{r o t}(t)}{c^{r o t}(t)} \equiv \frac{\tilde{c}^{r o t}(t)-c^{r o t}(t)}{c^{r o t}(t)}=\frac{\theta w(\tilde{R}-R)}{A+R \theta w} \quad \forall t \in[T, \bar{T}] .
$$

Since A is proportional to $w$, the wage rate can be factored out and eliminated from eq. [41]. Since $\partial A / \partial s>0$ and $\tilde{R}-R<0$, then $\partial\left[\frac{\Delta c^{r o t}(t)}{c^{r o t}(t)}\right] / \partial s>0$.

\section{Appendix D}

Partially differentiating eq. [19] with respect to $\rho$, reveals the sign of comparative statics depends on the sign of

$$
\frac{\partial g}{\partial \rho} \frac{\left(\bar{T} e^{(g-r) \bar{T}}\left(e^{(g-r) \bar{F}}-1\right)-\bar{F} e^{(g-r) \bar{F}}\left(e^{(g-r) \bar{T}}-1\right)\right)}{\left(e^{(g-r) \bar{F}}-1\right)^{2}} .
$$

As $\partial g / \partial \rho<0$, expression [42] is negative when

$$
\bar{T}\left(e^{(g-r) \bar{F}}-1\right) e^{(g-r) \bar{T}}>\bar{F} e^{(g-r) \bar{F}}\left(e^{(g-r) \bar{T}}-1\right) .
$$


For cases $g>r$ or $g<r$, expression [43] can be restated as

$$
\frac{\bar{T} e^{(g-r) \bar{T}}}{e^{(g-r) \bar{T}}-1}>\frac{\bar{F} e^{(g-r) \bar{F}}}{e^{(g-r) \bar{F}}-1} .
$$

For eq. [44] to hold, the derivative of the left-hand side of eq. [44] with respect to $\bar{T}$ must be negative, or

$$
(g-r) \bar{T}+1>e^{(g-r) \bar{T}},
$$

which cannot be true. Hence, eq. [42] is positive.

\section{Appendix E}

First, rewrite eq. [21] as

$$
\frac{\tilde{A}+\tilde{R} \theta w}{A+R \theta w}-1 \equiv \frac{a_{5} s+\tilde{R} \theta}{a_{6} s+R \theta}-1 .
$$

Now, note that eq. [19] can be compactly stated as

$$
a_{4}-1 \text {. }
$$

Finally, equate eqs [46]-[47] and solve for the unique, critical savings rate to confirm the proposition.

\section{Appendix F}

For the perfect foresight consumer with $g=0$, we start comparing $\frac{\varkappa(0)\left(e^{-r \bar{T}}-1\right)}{k(0)\left(e^{-r \bar{F}}-1\right)}-1$ (modified expression [19]) with $\frac{\tilde{k}(0)-k(0)}{k(0)}$ (expression [34]). Observe the latter is always negative. Hence, all we have to do is to prove modified eq. [19] is less than eq. [34]. Thus, we require

$$
\frac{\varkappa(0)\left(e^{-r \bar{T}}-1\right)}{k(0)\left(e^{-r \bar{F}}-1\right)}<\frac{\tilde{k}(0)}{k(0)} .
$$

Multiplying both sides of eq. [48] by $k(0)\left(e^{-r \bar{F}}-1\right)$, integrating-out $\varkappa(0)$ and $\tilde{k}(0)$, and collecting terms, finally produces the following inequality:

$$
\begin{gathered}
(1-\theta) w\left(e^{-r \bar{T}}-e^{-r(T+\bar{T})}-e^{-r \bar{F}}+e^{-r(T+\bar{F})}\right) \\
>\tilde{b}\left(e^{-r \bar{T}}-e^{-r(T+\bar{T})}-e^{-r \bar{F}}+e^{-r(T+\bar{F})}\right)
\end{gathered}
$$


We can divide both sides of eq. [49] by the bracketed expression. However first, we have to find out whether the expression is negative in sign.

Now, we rewrite the expression as $\Lambda_{1}-\Lambda_{2}$, where $\Lambda_{1} \equiv e^{-r \bar{T}}-e^{-r(T+\bar{T})}$, and $\Lambda_{2} \equiv e^{-r \bar{F}}-e^{-r(T+\bar{F})}$. Note that $\Lambda_{1}>0$. We also observe that $\Lambda_{2}$ is the same as $\Lambda_{1}$, but the only difference is $\bar{T}$ is replaced by $\bar{F}$. Since the latter is strictly greater than the former, all we need to know is how increasing $\bar{T}$ changes the value of $\Lambda_{1}$. Clearly, $\partial \Lambda_{1} / \partial \bar{T}=r\left(e^{-r(T+\bar{T})}-e^{-r \bar{T}}\right)$ is negative. Thus, $\Lambda_{1}>\Lambda_{2}$, meaning that expression [49] collapses to $(1-\theta) w>\tilde{b} \quad(\Leftarrow(1-\theta) w>b)$, which is true by assumption.

Now we consider the case when $g<0$ (or, $r<\rho)$. Using eqs [34] and [19], we need to prove that

$$
\frac{\varkappa(0)\left(e^{(g-r) \bar{T}}-1\right)}{k(0)\left(e^{(g-r) \bar{F}}-1\right)}<\frac{\tilde{k}(0)}{k(0)} .
$$

The latter inequality is the same as

$$
\varkappa(0)\left(e^{(g-r) \bar{T}}-1\right)>\tilde{k}(0)\left(e^{(g-r) \bar{F}}-1\right),
$$

or

$$
\begin{array}{r}
\left(\tilde{R} w \theta\left(e^{-r T}-e^{-r \bar{F}}\right)+w(1-\theta) e^{-r T}\left(e^{r T}-1\right)\right)\left(e^{(g-r) \bar{T}}-1\right) \\
>\left(\tilde{R} w \theta\left(e^{-r T}-e^{-r \bar{T}}\right)+w(1-\theta) e^{-r T}\left(e^{r T}-1\right)\right)\left(e^{(g-r) \bar{F}}-1\right)
\end{array}
$$

When $g<0$, the term $\left(e^{(g-r) \bar{F}}-1\right)$ is more negative than the term $\left(e^{(g-r) \bar{T}}-1\right)$. However, the term $\tilde{R} w \theta\left(e^{-r T}-e^{-r \bar{F}}\right)$ is positive but greater than the term $\tilde{R} w \theta\left(e^{-r T}-e^{-r \bar{T}}\right)$. Thus, eq. [52] may or may not hold. We clearly achieve the same sort of ambiguity in general when $g>0$.

Finally, for the rule-of-thumb consumer, compare eq. [21] with eq. [41]. Note that when $s>0, \tilde{A}<A$ (when $s=0, \tilde{A}=A=0$ ). The proof then follows.

\section{Appendix G}

Suppose

$$
k(0)-\varkappa(0)^{n e w} \geq 0 .
$$

Rewrite the left-hand-side of eq. [53] as 


$$
\begin{gathered}
-\left(R w \theta\left(e^{F r+\bar{F} r+r T}-e^{F r+r T+r \bar{T}}\right)+(w(1-\theta)-R w \theta)\left(e^{F r+\bar{F} r+r \bar{T}}-e^{\bar{F} r+r T+r \bar{T}}\right)\right) \\
\times \frac{e^{-F r-\bar{F} r-r T-r \bar{T}}}{r},
\end{gathered}
$$

or, as

$$
R w \theta\left(e^{F r+\bar{F} r+r T}-e^{F r+r T+r \bar{T}}\right)+(w(1-\theta)-R w \theta)\left(e^{F r+\bar{F} r+r \bar{T}}-e^{\bar{F} r+r T+r \bar{T}}\right) \leq 0 .
$$

The latter cannot be true since by assumption, $w(1-\theta)>\operatorname{Rw} \theta$, while $\bar{F}>\bar{T}$, and $F>T$. Thus, $\varkappa(0)^{\text {new }}>k(0)$.

\section{Appendix H}

Let $\beta \equiv \bar{F} / \bar{T}$. From eq. [28], we then have $F=\beta T$, while $\bar{F}=\beta \bar{T}$. Thus, eq. [32] can be restated as

$$
\tilde{A}^{\text {new }}=\frac{s(1-\theta) w\left(1-e^{\beta r T}\right)}{e^{\beta r(T-\bar{T})}-1} .
$$

Since $\beta>1$, eq. [56] implies that going from $A$ (see expression [11]) to $\tilde{A}^{\text {new }}$ is similar to increasing $r$. Thus, we can simply differentiate eq. [11] with respect to $r$ to check its sign. We have

$$
\frac{\partial A}{\partial r}=e^{r(T-\bar{T})} s(1-\theta) w \frac{\left(T\left(e^{r \bar{T}}-1\right)-\bar{T}\left(e^{r T}-1\right)\right)}{\left(e^{r(T-\bar{T})}-1\right)^{2}} .
$$

Clearly, eq. [57] is positive if $T\left(e^{r \bar{T}}-1\right)-\bar{T}\left(e^{r T}-1\right)$ is, and the latter indeed is greater than zero. ${ }^{12}$ Therefore, $\tilde{A}^{\text {new }}$ exceeds $A$.

\section{References}

Abdel-Ghany, M., and D. L. Sharpe. 1997. "Consumption Patterns among the Young-Old and Old-Old." The Journal of Consumer Affairs 31 (1):90-112.

Blackorby, C., W. Bossert, and D. J. Donaldson. 2005. Population Issues in Social Choice Theory, Welfare Economics, and Ethics. Cambridge: Cambridge University Press.

12 The proof is omitted for the sake of brevity, but is straightforward and available upon request. 
Boyle, M. 2013. “Aging Boomers Stump Marketers Eyeing \$15 Trillion Prize.” Bloomberg Business (September 17).

Bradshaw, D. 2015. “Ageing Population in China Creates Business Opportunities.” The Financial Times Limited (March 29).

Brunner, J. K. 2002. “Welfare Effects of Pension Finance Reform.” Johannes Kepler University of Linz Working Paper.

Campbell, J. Y., and N. Gregory Mankiw. 1989. "Consumption, Income and Interest Rates: Reinterpreting the Time Series Evidence." In NBER Macroeconomics Annual 1989, edited by O. J. Blanchard and S. Fischer, 185-216. Cambridge, MA: MIT Press.

Campbell, J. Y., and N. Gregory Mankiw. 1990. "Permanent Income, Current Income, and Consumption." Journal of Business and Economic Statistics 8 (3):265-79.

Cremer, H., P. De Donder, D. Maldonado, and P. Pestieau. 2008. "Designing a Linear Pension Scheme with Forced Savings and Wage Heterogeneity." International Tax and Public Finance 15 (5):547-62.

Diamond, P. A., and P. R. Orszag. 2005. Saving Social Security: A Balanced Approach, Revised Edition. Washington, D.C.: The Brookings Institution.

Docquier, F. 2002. "On the Optimality of Public Pensions in an Economy with Life-Cyclers and Myopes." Journal of Economic Behavior and Organization 47 (1):121-40.

Feigenbaum, J. 2008. “Can Mortality Risk Explain the Consumption Hump?” Journal of Macroeconomics 30 (3):844-72.

Feigenbaum, J., F. Caliendo, and E. Gahramanov. 2011. "Optimal Irrational Behavior." Journal of Economic Behavior and Organization 77 (3):285-303.

Feigenbaum, J., E. Gahramanov, and X. Tang. 2013a. “Is It Really Good to Annuitize?” Journal of Economic Behavior and Organization 93:116-40.

Feigenbaum, J., E. Gahramanov, and X. Tang. 2013b. "Can High Discount Rates Increase Capital Accumulation?" Utah State University Working Paper.

Feldstein, M. 1985. "The Optimal Level of Social Security Benefits." Quarterly Journal of Economics 100 (2):303-20.

Findley, S. T., and F. Caliendo. 2007. "OutSMarTing the Social Security Crisis." Public Finance Review 35 (6):647-68.

Findley, S. T., and F. N. Caliendo. 2009. "Short Horizons, Time Inconsistency, and Optimal Social Security." International Tax and Public Finance 16 (4):487-513.

Findley, S. T., and F. N. Caliendo. 2010. "Does It Pay to Be SMarT?" Journal of Pension Economics and Finance 9 (3):321-44.

Hurst, E., and P. Willen. 2007. "Social Security and Unsecured Debt." Journal of Public Economics 91 (7-8):1273-97.

Huseby, R. 2012. "Sufficiency and Population Ethics." Ethical Perspectives 19 (2):187-206.

İmrohoroğlu, A., S. İmrohoroğlu, and D. H. Joines. 2003. "Time-Inconsistent Preferences and Social Security." Quarterly Journal of Economics 118 (2):745-84.

Kaplow, L. 2006. "Myopia and the Effects of Social Security and Capital Taxation on Labor Supply.” NBER Working Paper No. 12452.

Kronenberg, T. 2009. "The Impact of Demographic Change on Energy Use and Greenhouse Gas Emissions in Germany.” Ecological Economics 68 (10): 2637-45.

Lacomba, J. A., and F. Lagos. 2006. "Population Ageing and Legal Retirement Age." Journal of Population Economics 19 (3):507-19. 
Lusardi, A. 1999. “Information, Expectations, and Savings for Retirement.” In Behavioral Dimensions of Retirement Economics, edited by H. Aaron, 81-115. Washington, DC: Brookings Institution Press and Russell Sage Foundation.

Modigliani, F. 1986. "Life Cycle, Individual Thrift, and the Wealth of Nations." American Economic Review 76 (3):297-313.

NSW Government. 2012. NSW Ageing Strategy. Sydney: Department of Family and Community Services, Office for Ageing.

Piketty, T. 2014. “Economics of Inequality." Paris School of Economics Lecture Notes.

Thaler, R. H. 1994. "Psychology and Savings Policies." American Economic Review 84 (2):186-92.

Thaler, R. H., and S. Benartzi. 2004. "Save More Tomorrow ${ }^{\mathrm{TM}}$ : Using Behavioral Economics to Increase Employee Saving.” Journal of Political Economy 112 (1):S164-S187.

The Rockefeller Foundation. 2014. Rockefeller Millennials Survey. Global Strategy Group. Wong, W.-K. 2012. "Consumption Response to Government Transfers: Behavioral Motives Revealed by Savers and Spenders.” Contemporary Economic Policy 30 (4):489-501. 